\title{
Sensitivity of state-of-the-art and high efficiency crystalline silicon solar cells to metal impurities
}

\author{
Gianluca Coletti* \\ ECN Solar Energy, Westerduinweg 3, Petten NL-1755 LE, The Netherlands
}

\begin{abstract}
For the first time, the sensitivity to impurities of the solar cell conversion efficiency is reported for a state-of-the-art (i.e., 18\%) and advanced device architecture (i.e., 23\%). The data are based on the experimental results obtained in the CrystalClear project for the state-of-the-art cell process and extrapolated to a device with excellent front and rear surface passivation. Both device structures are not assumed to work in low injection level as several studies assumed before, but real operating conditions are considered. This is a fundamental difference with the past and required for modeling future high efficiency devices. The impurity with highest impact is $\mathrm{Ti}$, followed by $\mathrm{Cu}, \mathrm{Cr}, \mathrm{Ni}$ and $\mathrm{Fe}$, which form together a group two order of magnitude less sensitive than the former. In high efficiency devices, a large reduction of the impurity impact is visible for impurities with large capture cross-section ratio like Fe, which reduces its relative difference in comparison with, for example, $\mathrm{Cr}$, which has a small capture cross-section ratio. In general, advanced devices will be more sensitive to the impurity content than the state-of-the-art cell design. This effect is partly compensated by a reduction of the substrate thickness. The impurity sensitivity as function of the substrate thickness is reported. Copyright (C) 2012 John Wiley \& Sons, Ltd.
\end{abstract}

\section{KEYWORDS}

impurities; feedstock; polysilicon; solar-grade silicon; silicon; solar cell; efficiency; degradation

\author{
* Correspondence \\ Gianluca Coletti, ECN Solar Energy, Westerduinweg 3, Petten, NL-1755 LE The Netherlands. \\ E-mail: coletti@ecn.nl
}

Received 11 October 2011; Revised 28 January 2012; Accepted 10 February 2012

\section{INTRODUCTION}

Energy supply from photovoltaics (PV) will be dominating in the next decades. The European commission sets a target of $20 \%$ of energy production to come from renewable sources by 2020, and solar energy will have a large role [1]. Currently, crystalline silicon solar cells contribute to over $80 \%$ of the total supply of PV modules. It is therefore reasonable that the technology, which will most probably dominate the supply of PV in the years to come, will be based on crystalline silicon substrates. The substrate characteristics, which are the topic of this letter, should not limit the solar cell conversion efficiency and should enable architectures suited to obtain high efficiency and optimized cost/performance ratio. In Figure 1, the sensitivity of the efficiency towards the diffusion length for a state-of-the-art and an advanced device (as defined in Table I) is reported. It shows that for a state-of-the-art device, the relative efficiency saturates $(>98 \%)$ for diffusion lengths three times above the wafer thickness. This is because the surface passivation is limiting the effective lifetime of the device. An advanced device with excellent surface passivation is still sensitive to increases in the diffusion length in this range. This shows that the sensitivity to impurities of crystalline silicon solar cells will largely increase for higher efficiency devices, which is the topic of this letter.

\section{BACKGROUND AND COMPARISON BETWEEN WESTINGHOUSE AND CRYSTALCLEAR STUDIES}

The minority carrier diffusion length of crystalline silicon is intrinsically limited by Auger and band-to-band recombination which cannot be avoided [2]. A substrate with resistivity of $1 \Omega \mathrm{cm}$ and injection level (operation condition) of $\Delta \mathrm{n}=1 \times 10^{15} \mathrm{~cm}^{-3}$ is ideally limited by a diffusion length 


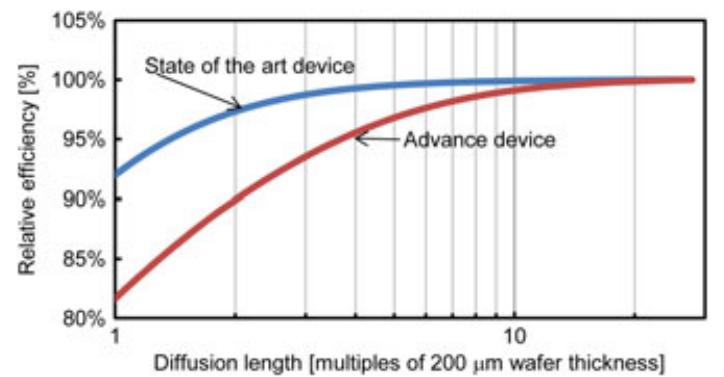

Figure 1. Relative efficiency as function of the diffusion length for a state-of-the-art and advanced device. The diffusion length is reported in unit of wafer thickness $(200 \mu \mathrm{m})$.

of about $3 \mathrm{~mm}$. In practice, substrates are dominated by recombination via, for example, impurities, dislocations and grain boundaries. The impact of impurities on solar cell device has been extensively investigated within the framework of the CrystalClear project [3] (CC study). The effect of several impurities known for their severe impact on device performances was studied by growing multicrystalline silicon ingots with intentional contamination of a known amount of each element. Results of this work were reported in [4]. The groundbreaking research performed in the 1970s and 1980s by Westinghouse Corp[5] (WH study) is still used as a reference for the effect of impurities on solar cell performance despite the large amount of research produced later [6-11]. In this letter, for the first time, the updated efficiency degradation curves for several impurities based on the recent experimental data from the $\mathrm{CC}$ study are reported.

One of the most important differences between the WH and $\mathrm{CC}$ investigations is that the $\mathrm{CC}$ data refers to a modern, state-of-the-art p-type industrial screen-print solar cell process[4], and that the impact of impurities is investigated on directionally solidified multicrystalline material, as opposed to Czochralski silicon ingots. In the CC study, the direct relationship between impurities in the feedstock and the final solar cell performance is analyzed. This provides a very straightforward and meaningful interpretation, especially addressed at specifying feedstock impurity tolerances. The CC conclusions, therefore, do not rely on impurity analysis or estimated segregation coefficients. By comparison, the previous Westinghouse studies rely on the estimated effective segregation coefficient to determine the impurity concentration in the wafers and relate the latter with the solar cell performance.

In addition, in this study, the total ingot height, corresponding to $100 \%$ of the melt weight, was used for the experiments and the evaluation, as opposed to only one ingot section, analyzed in the WH project. Therefore, each ingot provides a broad range of impurity concentration levels in the wafers. For example, at $90 \%$ of the ingot height, the impurity concentration for the majority of metal impurities is about 10 times higher than that in the bottom because of the segregation of impurities. To gain insight into the impurity contamination level allowed, it is of great importance to relate the impact of impurities to the fully usable fraction of the ingot as carried out in the CC approach. An example of this is reported in Figure 2, where a degradation of the solar cell performance from the bottom to the top of the ingots is visible. From this example, it could be concluded that $90 \%$ of the ingot height can be considered an indicative limit to calculate the impact of impurities. According to the normal freezing equation, at an ingot height of $99 \%$, the impurity concentration is 100 times higher than that in the bottom, therefore, data extrapolation to different ingot height is relatively straightforward.

Another major difference between $\mathrm{CC}$ and $\mathrm{WH}$ is in the model used for the data analysis. The WH study relies on an analytical solution of the transport equations, which results in a device illuminated by monochromatic light (not full AM1.5 solar spectrum) and assumed to be diffusion length limited. In this way, WH avoided the direct (and time-consuming) measurements of diffusion length relying only on the short circuit current measurements (which in this assumption is directly related to the diffusion length). The CC study used a software tool (PC1D) [12] for the numerical solution of transport equations and the full AM1.5 solar spectrum. The internal quantum efficiency was used together with the IV-parameters to model the solar cell with PC1D, considering both the bulk and emitter regions [4]. In both these studies and other studies $[8,11,13]$ as well, the device was considered operating in low injection level conditions.

In this letter, we illustrate the developments carried out to model the device at the operating injection level (no low injection level assumption, see Figure 3), therefore, also opening the possibility to model a future device with excellent surface passivation and with higher efficiency. To our knowledge, this approach has not been used before.

Table I. Device model parameters, for a p-type substrate with an n-type front emitter. Parameters are reported for a state-of-the-art and an advanced solar cell design.

\begin{tabular}{lcccccc}
\hline Parameter device & Resistivity $(\Omega \mathrm{cm})$ & Thickness $(\mu \mathrm{m})$ & Emitter $(\Omega / \square)$ & Front SRV & Rear SRV & Rear reflector $(\%)$ \\
\hline State of the art 2010* & 1.1 & 200 & 60 & $1 \times 10^{5} \mathrm{~cm} / \mathrm{s}$ & $350 \mathrm{~cm} / \mathrm{s}$ & 67 \\
& 1.1 & 100 & 100 & $\begin{array}{l}400 \mathrm{fA} / \mathrm{cm}^{2} \\
2000 \mathrm{~cm} / \mathrm{s}\end{array}$ & $\begin{array}{l}400 \mathrm{fA} / \mathrm{cm}^{2} \\
10 \mathrm{~cm} / \mathrm{s}\end{array}$ & 85 \\
Advanced & & & & $20 \mathrm{fA} / \mathrm{cm}^{2}$ & $20 \mathrm{fA} / \mathrm{cm}^{2}$ & \\
\end{tabular}

*Typical values for a multicrystalline p-type solar cell 


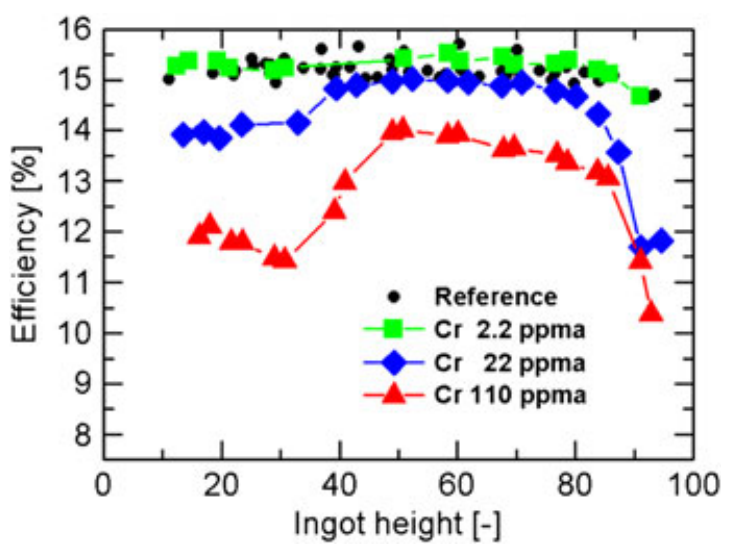

Figure 2. The product of short-circuit current and open-circuit voltage versus position in the ingot from the bottom, for the ingots contaminated with chromium. The model used is based on the degradation of the base-bulk recombination. The parameter fitting was carried out on the ingot with 22 ppma of chromium, with excellent result. The model has been validated using the ingots with 2.2 and 110 ppma.

\section{MODEL}

This letter addresses the impact on current and future device architectures of main metal impurities present in commercial silicon substrates. In the model developed here, the effect of operational injection level, which is important for high efficiency devices, is taken into account. Therefore, we need to know both the majority and minority carrier recombination parameters. In previous publications, the solar cell is always assumed to work in low injection level conditions. This is a good assumption for state-ofthe-art industrial p-type multicrystalline silicon solar cells based on screen print, $\mathrm{SiN}_{\mathrm{x}}$ firing through and Al-BSF process. This assumption is no longer valid in high efficiency solar cells and when substrates from improved crystallization method are employed (e.g., for the growth of multicrystalline silicon ingots).
In this model, the substrate is only assumed to be limited by Auger and band-to-band recombination in addition to the impurity to study. Therefore, the model can be adapted to any type of material monocrystalline or multicrystalline, with different levels of other defects as crystallographic defects for multicrystalline wafers and boron-oxygen (BO) complex for monocrystalline wafers.

The assumptions for the formulation of this model are listed as follows:

(i) The impurity distribution along the ingot height scales according to the normal freezing equation

$$
C_{\mathrm{s}}=k_{\mathrm{eff}} C_{0}(1-f)^{\left(k_{\mathrm{eff}}-1\right)}
$$

where $C_{\mathrm{s}}$ is the total impurity concentration in the solid, $C_{O}$ is the starting impurity concentration in molten silicon or in the feedstock, $k_{\text {eff }}$ is the effective segregation coefficient and $f$ is the solidified fraction. Impurities segregate towards the last solidified part of ingots. In this letter, we consider the effect at $90 \%$ of the ingot height because at lower height, the impurity impact will be lower, and we are interested here in taking into account the usable fraction of an ingot when considering the impurity impact. Therefore, $C_{\mathrm{s}}$ becomes $C_{\mathrm{s}}=10 \times k_{\mathrm{eff}} C_{0}$ for $f=90 \%$ and $\mathrm{k}_{\mathrm{eff}}<1$, 10 times larger than for $f=0 \%$ (ingot bottom).

(ii) The concentration of electrically active impurities $\left(\mathrm{N}_{\mathrm{t}}\right)$ is proportional to the total concentration of impurities $\left(\mathrm{C}_{s}\right)$,

$$
N_{t}=\alpha C_{\mathrm{s}}
$$

(iii) The minority carrier lifetime in the base-bulk or in the emitter can be described by the Shockley-Read-Hall

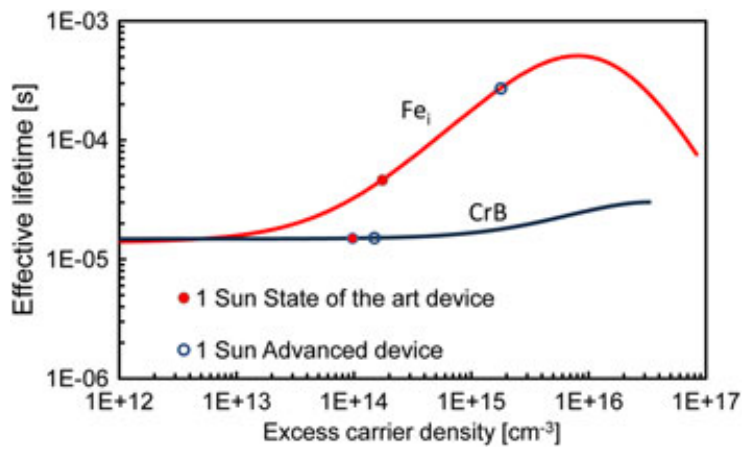

Figure 3. Lifetime versus excess carrier density for interstitial $\mathrm{Fe}$ and $\mathrm{CrB}$. The concentration of $\mathrm{Fe}_{i}$ and $\mathrm{CrB}$ are chosen in order to have the same low injection level lifetime $\left(5 \times 10^{11} \mathrm{~atm} / \mathrm{cm} 3\right.$ and $3 \times 10^{11} \mathrm{~atm} / \mathrm{cm} 3$, respectively). The curve for Fe $\mathrm{e}_{\mathrm{i}}$ is much steeper than for $\mathrm{CrB}$ owing to the high capture cross section ratio of $\mathrm{Fe}_{\mathrm{i}}$ in comparison to $\mathrm{CrB}$. The solar cell operational injection level at 1 sun is reported for a state-of-the-art and advanced devices as defined in Table I. In case of $\mathrm{Fe}_{\mathrm{i}}$, note the increased injection level and, therefore, lifetime at 1 sun in an advanced device owing to the steep lifetime-injection level dependence. This is not the case

for $\mathrm{CrB}$, where the relatively flat lifetime-injection level dependence does not increase considerably the injection level at 1 sun. 
(SRH) theory [14]. No assumption has been made on solar cells working in low injection level in the base.

(iv) The effect of impurities on the solar cell is that of increasing the recombination in the base and in the emitter regions. The solar cell performance as a function of base and emitter lifetime has been modeled using PC1D [12]. The devices modeled are a state-of-the-art 2010 design and an advanced design (see Table I for the definitions).

The assumption in (i) has been verified by neutron activation analysis on several samples as reported in Reference [15]. The electrical activity of a specific impurity is influenced by phenomena such as precipitation, gettering and hydrogenation, which are process dependent. Therefore, the proportionality constant $(\alpha)$ is element and process dependent and is a global parameter because it correlates the electrical activity of an impurity at solar cell level with the total solid impurity concentration $C_{\mathrm{s}}$ in the wafer.

The device minority carrier lifetime in (iii) is assumed to be dominated by a single impurity. Therefore, the Auger and band-to-band recombination are the only second-order limiting factors considered:

$$
\frac{1}{\tau_{\text {bulk }}}=\frac{1}{\tau_{\text {impurity }}}+\frac{1}{\tau_{\text {Auger }+ \text { radiative }}}
$$

This means that in the model, other defects like crystallographic defects or other impurities are neglected because we are studying a single defect (here an impurity) per time. In monocrystalline and multicrystalline silicon wafers, one could argue that, especially at low impurity concentration, other defects contribute to the recombination as well and cannot be neglected. This is the case, for example, of BO complex and crystallographic defects dominant in monocrystalline and multicrystalline silicon device, respectively. When they dominate the recombination, a specific term relative to their recombination should be added to Equation (3). In this case, the figures on the impurity sensitivity reported in this letter are restrictive because the sensitivity towards impurity concentration reduces in the presence of other dominating defects.

In this model, we use the parameters inferred on intentionally contaminated ingots with iron, titanium, chromium, nickel and copper in p-type silicon reported in [4] (see Table II).
The assumption is that impurity segregation in the ingot, electrical activity and recombination parameters are similar in both the devices modeled. This is reasonable because in [4], the effect of the impurities have been decoupled from other sources of recombination, such as crystallographic defects present in uncontaminated reference material. Therefore, the parameters inferred are relative to the only effect of the single impurity added. On the other hand, the effect of the additional crystallographic defects caused by the interaction between impurity and crystal growth, observed at high impurity concentrations, is intrinsically taken in consideration when the experimental data were fitted in the model in [3]. We assume that the impact of added impurity results in a recombination that is proportional to the concentration of the added impurity. Therefore, the value of electrical activity $\alpha$ is the same (for each specific impurity) for any impurity concentration considered (assumption (ii) in the model).

In assumption (iii), the effect of the lifetime-injection level dependence [16] is taken into account. This is especially relevant for impurities such as interstitial $\mathrm{Fe}$ ( $\mathrm{Fe}_{\mathrm{i}}$ is dominant under illumination condition), which has a larger minority carrier capture cross section than the majority rate (ratio $\sigma_{\mathrm{n}} / \sigma_{\mathrm{p}} \sim 500$ ). In Figure 3 , this effect is visible on the lifetime versus excess carrier density curves. For $\mathrm{Fe}_{\mathrm{i}}$, the curve is very steep towards higher injection level in comparison to the one for $\mathrm{CrB}(\mathrm{CrB}$ is dominant under cell operation condition than interstitial $\mathrm{Cr}$ ). This is because for the latter, the carrier capture cross section ratio is small. By improving the surface passivation, as in advanced devices, the injection level, corresponding to one-sun illumination, increases from the low injection level as the surface recombination reduces. At higher injection levels, the lifetime is controlled by both minority and majority capture rates, therefore, for $\mathrm{Fe}_{\mathrm{i}}$, the lifetime increases considerably in comparison to $\mathrm{CrB}$ (see Figure 3 ).

The operational injection level for a solar is determined by the total recombination in the device. Therefore, the injection level increases also as a consequence of the reduction of other bulk defects, as is seen in improved crystallization techniques. The increased injection level has a large impact on the solar cell performance because the bulk lifetime at which the solar cell operates is higher.

In case of copper, it was not possible in [4] to estimate its electrical activity. The reason is that there is no agreement in the recombination parameters for both electron and holes,

Table II. Segregation coefficient, recombination parameter and electrical activity of impurities used for the modeling.

\begin{tabular}{|c|c|c|c|c|}
\hline & Effective segr. coeff. [4] & Minority carrier capture cross section & & Electrical activity [4] \\
\hline Impurity & $k_{\text {eff }}[-]$ & $\sigma_{\mathrm{n}}\left[\mathrm{cm}^{2}\right]$ & Ec-Et & $\alpha[\%]$ \\
\hline $\mathrm{Cr}$ & $3.1 \times 10^{-6}$ & $\begin{array}{l}\sigma_{\mathrm{nCrB}}=4.5 \times 10^{-15} \\
\sigma_{\mathrm{pCrB}}=1.1 \times 10^{-14}\end{array}$ & $0.844[18]$ & 5.90 \\
\hline $\mathrm{Fe}$ & $1.5 \times 10^{-5}$ & $\begin{array}{l}\sigma_{\mathrm{nFei}}=4 \times 10^{-14} \\
\sigma_{\mathrm{pFei}}=7 \times 10^{-17}\end{array}$ & $0.745[19]$ & 0.11 \\
\hline $\mathrm{Ti}$ & $3.5 \times 10^{-5}$ & $\begin{array}{l}\sigma_{\text {nTidd }}=1.5 \times 10^{-15} \\
\sigma_{\text {pTidd }}=3.7 \times 10^{-17}\end{array}$ & $0.865[20]$ & 93 \\
\hline
\end{tabular}


therefore, the lifetime is modeled at same operation condition as a present device [4] and therefore in low injection level. The emitter is always considered in low injection level owing to the high doping level. In addition to the SRH recombination caused by the impurity under investigation, Auger and band-to-band recombination are considered in the model as the only additional recombination mechanisms because they are a fundamental limit. These recombinations become relevant especially at low resistivity, high injection levels and in general at very low defects density.

Impurities like titanium, chromium and iron have been reported to reduce the bulk recombination (diffusion length), therefore, the SRH theory is used to model the bulk lifetime in PC1D. Contrarily, nickel only affects the emitter recombination [4], therefore, the emitter lifetime is used in the PC1D model. On the other hand, $\mathrm{Cu}$ affects both bulk and emitter recombination, therefore, both recombinations have been used to model its effect in PC1D [4]. For the explanation and the description of the modeling fitting parameters, we refer to [4].

\section{RESULTS AND DISCUSSION}

The device model parameters, for the state-of-the-art 2010 design with a p-type substrate and an n-type front emitter, are reported in Table I. In case of absence of bulk defects (device limited only by Auger and band-to-band recombination), an efficiency of about $17.5 \%$ is obtained. In Figure 4, the solar cell efficiency calculated at $90 \%$ of the ingot height is plotted versus the impurity concentration in the feedstock.

Note that the efficiency is very sensitive to the presence of $\mathrm{Ti}$. $\mathrm{Cu}, \mathrm{Cr}, \mathrm{Ni}$ and $\mathrm{Fe}$, which form together a group, two orders of magnitude less sensitive than the former. The reason is that $\mathrm{Ti}$, being a slow diffuser, has small or negligible relaxation and segregation gettering [17] effectiveness as expressed from its electrical activity close to one (100\% active, see Table II). On the other hand, Fe, but also $\mathrm{Cu}$ and $\mathrm{Ni}$, being fast or relatively fast diffusing

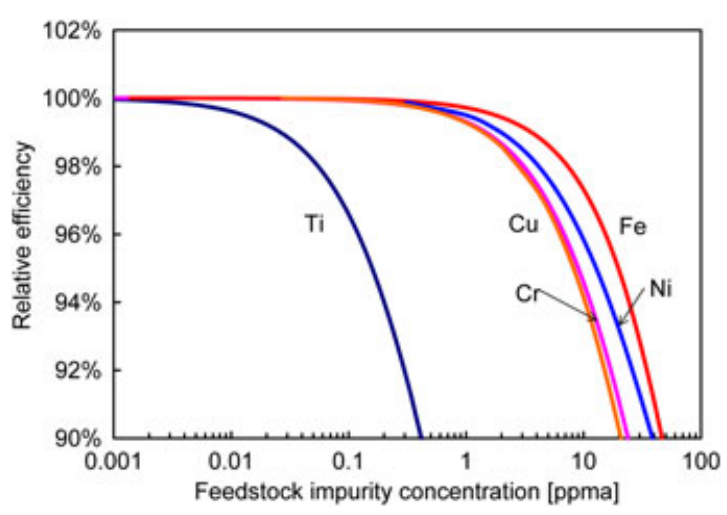

Figure 4. Relative efficiency at $90 \%$ of the ingot versus impurity conversion in the feedstock. Device is a state-of-the-art cell process with $17.5 \%$ cell efficiency limited by Auger and band-to-band recombination with $200 \mu \mathrm{m}$ cell thickness. impurities, are more sensitive to gettering. The effect of nickel is to increase the recombination in the emitter, and no effect was reported on the bulk recombination [4]. However, in the case of a device limited by Auger and band-to-band recombination (e.g., negligible presence of other defects), the lifetime is much higher than in the experimental case studied, and therefore, the effect of nickel can potentially extend to the bulk as well, similarly to the case of $\mathrm{Cu}$. Therefore, the effect of $\mathrm{Ni}$ in this model should be considered as an upper limit.

Many studies report that, despite of a high level of metal contamination, similar solar cell performance can be achieved as that obtained using electronic grade purity feedstock $[4,7,21,22]$. These studies have been carried out for a state-of-the-art industrial type process for both the solar cell and the ingot growth procedures. It is expected that with improved crystallization and solar cell processing, the sensitivity towards impurity will increase. The feedstock purity limits are therefore a strong function of the device architecture and substrate material quality. We are interested to estimate the impact of impurities on a device that meets the characteristics [23] of a future solar cell. Therefore, we model a device, which is not limited by front and rear surface passivation, meaning cells with excellent surface passivation. Most likely, the substrate thickness will be reduced as shown by the steep trend in the last several years. In Table I, the device model parameters for excellent surfaces and substrate thickness of $100 \mu \mathrm{m}$ are reported. In case of absence of bulk defects (device limited only by Auger and band-to-band recombination), an efficiency of about $23 \%$ is obtained.

We do not know the impact of $\mathrm{Ni}$ and $\mathrm{Cu}$ on such advanced emitter, consequently, they cannot be modeled here. Note that the improved surface passivation might increase considerably the impact of those impurities that affect the emitter recombination (such as $\mathrm{Cu}$ and $\mathrm{Ni}$ ), therefore, effort should be put in reducing their effect (e.g., by dedicated etching and cleaning) and/or in reducing their presence.

To model the minority carrier lifetime for this device, we use the same assumption and parameters for the state-of-the-art device considered here. This means that the impurity global electrical activity is considered the same as on the state-of-the-art device, and that proportionality is kept between total impurity concentration and active impurity concentration. In Figure 5, the efficiency at $90 \%$ of the ingot height for this device is plotted versus the impurity concentration in the feedstock. One could note that the relative difference between $\mathrm{Cr}$ and $\mathrm{Fe}$ changed (Figure 6). This is because in this advanced device, and especially at relatively low impurity concentration, the solar cells do not operate anymore at low injection level. Actually, the smaller the impurity level, the higher the injection level. The effect of impurities like Fe, with a high capture cross section ratio $\sigma_{\mathrm{n}} / \sigma_{\mathrm{p}}$, therefore, with a steep lifetime injection level dependence, benefit from an increasing lifetime because the injection level increases [24]. This is also mirrored in a more steep sensitivity curve in the case of $\mathrm{Fe}$ (i.e., from right to the left of Figure 5), this 


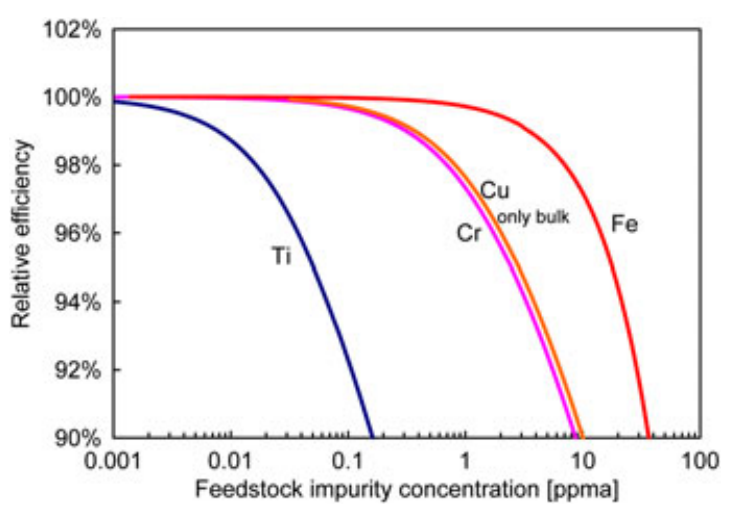

Figure 5. Relative efficiency at $90 \%$ of the ingot versus impurity concentration in the feedstock. Device has a cell efficiency of $23 \%$ limited by Auger and band-to-band recombination with $100 \mu \mathrm{m}$ cell thickness.

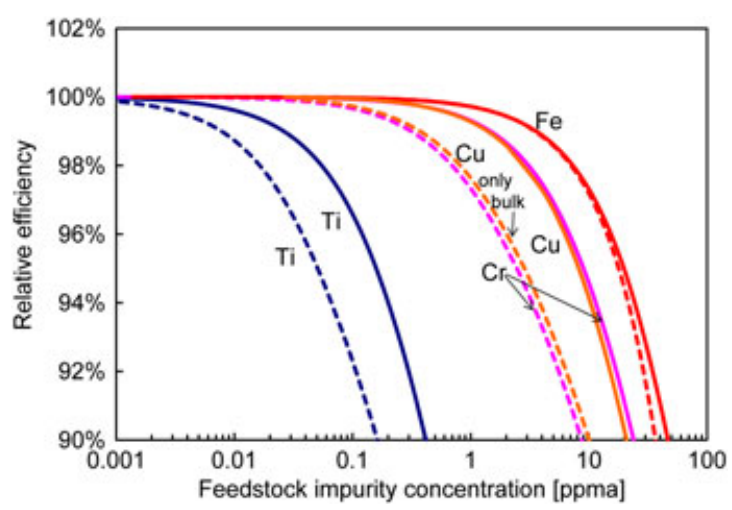

Figure 6. Relative efficiency degradation curve at $90 \%$ of the ingot versus impurity concentration in the feedstock for the state-of-the-art $200-\mu \mathrm{m}$ thick device (full lines) and for advanced $100-\mu \mathrm{m}$ thick device (dash lines).

is due to the increase in the injection level when the amount of $\mathrm{Fe}$ reduces. This reduced sensitivity for $\mathrm{Fe}$ partly compensates the requirements of higher lifetime in advanced devices, and together with the reduced substrate thickness make the impact of Fe similar in both device architectures. Instead, $\mathrm{Cr}$ with small capture cross sections ratio (flat injection level dependency) is less sensitive to this effect, and it impact increases. In case of $\mathrm{Cu}$, the only effect on bulk lifetime is taken into account.

Note that the operational injection level is determined by the total recombination in the device. The presence of other defects like other impurities, BO complexes and crystallographic defects reduce the operation injection level and therefore the operating lifetime. This means that in the presence of these other defects, the cell performance sensitivity towards Fe will increase.

Note that in general, the efficiency sensitivity towards impurity increases significantly in a high efficiency device despite the reduction of half the base thickness that only partly compensates the increased sensitivity. The sensitivity to the wafer thickness is shown in Figure 7, where impurity sensitivity curves are plotted for substrates as thick as 50,100 and $200 \mu \mathrm{m}$.

The efficiency degradation curves can be used to define the relative impact of impurities. This is relevant for feedstock and ingot producers to define roadmap and technologies for improvement programs and cost reduction. It is important to note that interaction of defects can cause a non-linear behavior, and the combination of dominating impurities complicates the analysis and the estimate in the specific case.

This assumption on the electrical activity means that in the future device, the same optimum gettering and hydrogenation of current device is used so as to have a realistic model. Depending on the nature of the device architecture implemented (for example, hetero-junction where the temperature employed and gettering effect is low), the electrical activity can increase, and consequently, the impact of impurities on the device increases as well. On the other hand R\&D effort to improve the wafer quality, optimize gettering and hydrogenation and reduce the impact on emitter recombination will definitely be implemented in the future solar cell process. Therefore, the predictions of this work should be considered conservative. Note that a more effective gettering and hydrogenation, which results in a 10 times reduction of electrical activity will give similar efficiency as 10 times less impurities concentration (without considering the injection level dependence effect), shifting the sensitivity curve in Figure 5, leftwards.

\section{CONCLUSIONS}

For the first time, the sensitivity to impurities of the solar cell conversion efficiency is reported for state-of-the-art and advanced device architectures. In a state-of-the-art

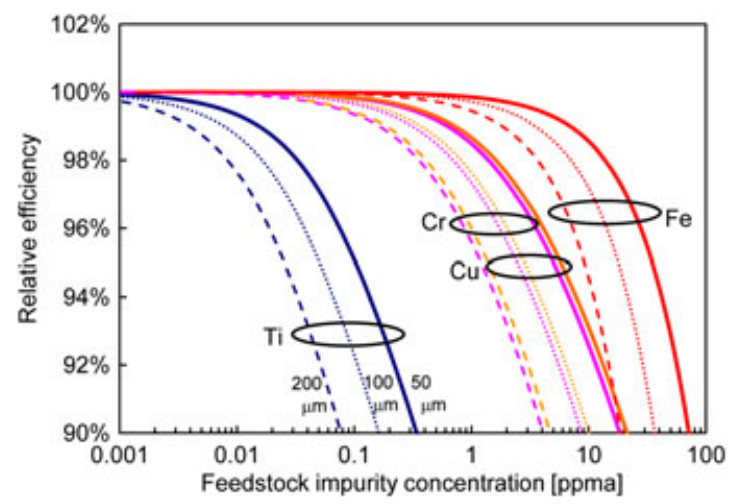

Figure 7. Relative efficiency at $90 \%$ of the ingot versus impurity concentration in the feedstock. Device has a cell efficiency of $23 \%$ limited by Auger and band-to-band recombination with 50, 100 and $200 \mu \mathrm{m}$ cell thickness. Cu was plotted considering the impact on the diffusion length only. 
device, $\mathrm{Ti}$ is the most impacting impurity, followed by $\mathrm{Cu}$, $\mathrm{Cr}$, Ni and $\mathrm{Fe}$, which form together a group, two order of magnitude less sensitive than the former. Contrarily to previous modeling, it is not assumed that the device is working in low injection level conditions, but in real operating conditions. This is of fundamental importance for modeling high efficiency devices. In high efficiency devices, as opposite to state of the art, a large reduction of the impurity impact is visible for impurities having large capture cross section ratio such as Fe. This reduction partly compensates the requirements of higher lifetime in advanced devices. By contrast, the sensitivity of $\mathrm{Cr}$ increases because it is not compensated by higher lifetime owing to the small capture cross section ratio.

In general, future devices will be more sensitive to impurity content than state-of-the-art devices. This effect could be partly compensated by reducing the base thickness as shown.

The data provided in this study and the efficiency degradation curves for the different cases studied make it possible to calculate impurity acceptable levels. Specifications of acceptable levels are function of acceptable efficiency, cell architecture and process technology, crystallization method, substrate defect content and wafer thickness. In conclusion, the data reported allows, on one side, to focus R\&D efforts towards a selective control of the feedstock impurity content and, on the other side, to perform sensitivity analysis at different stages of the solar cell manufacturing, keeping in mind the different impurity cell performance sensitivities.

\section{ACKNOWLEDGEMENT}

This work was partly funded by the Dutch Ministry of Economic Affairs, Agriculture and Innovation.

\section{REFERENCES}

1. http://www.setfor2020.eu

2. Kerr MJ, Cuevas A. Journal of Applied Physics 2002; 91: 2473 .

3. Sinke WC, van Hooff WMG, Coletti G, Ehlen BE, Hahn G, Reber S, John J, Beaucarne G, van Kerschaver E, de Wild-Scholten MJ, Metz A. Wafer-based crystalline silicon modules at 1 Euro/Wp: final results from the CrystalClear integrated project. 24th European Photovoltaic Solar Energy Conference and Exhibition, Hamburg, Germany, 21-25 September, 2009; 845.

4. Coletti G, Bronsveld PCP, Hahn G, Warta W, Macdonald D, Ceccaroli B, Wambach K, Le Quang N, Fernandez JM. Impact of metal contamination in silicon solar cells. Advanced Functional Materials 2011; 21(5): 879-890 and reference therein.
5. Davis JR, Rohatgi A, Hopkins RH, Blais PD, Rai-Choudhury P, McCormick JR, Mollenkopf HC. IEEE Transactions on Electron Devices 1980; 27: 677 and reference therein.

6. Pizzini SBS, Acciarri M, Binetti S. From electronic grade to solar grade silicon: chances and challenges in photovoltaics. Physica Status Solidi A 2005; 202: 2928; Pizzini S. Towards solar grade silicon: challenges and benefits for low cost photovoltaics. Solar Energy Materials and Solar Cells 2010; 94: 1528.

7. Riepe S, Reis IE, Kwapil W, Falkenberg MA, Schön J, Behnken H, Bauer J, Kreßner-Kiel D, Seifert W, Koch W. Research on efficiency limiting defects and defect engineering in silicon solar cells-results of the German research cluster SolarFocus. Physica Status Solidi C 2011; 8: 733

8. Geerligs LJ, Manshanden P, Wyers GP, Øvrelid EJ, Raaness OS, Waernes AN, Wiersma B. Proceedings of the 20th EUPVSEC, Barcelona, Spain, 2005; 619, (unpublished).

9. Dubois S, Palais O, Pasquinelli M, Martinuzzi S, Jassaud $\mathrm{C}$, Rondel N. Influence of iron contamination on the performances of single - crystalline silicon solar cells: computed and experimental results. Journal of Applied Physics 2006; 100: 024510; Dubois S, Palais O, Ribeyron PJ, Enjalbert N, Pasquinelli M, Martinuzzi S. Effect of intentional bulk contamination with iron on multicrystalline solar cell properties. Journal of Applied Physics 2007; 102: 083525.

10. Macdonald D, Mäckel H, Cuevas A. Applied Physics Letters 2006; 88: 092105.

11. Hofstetter J, Lelièvre JF, del Canizo C, Luque A. Acceptable contamination levels in solar grade silicon: from feedstock to solar cell. Materials Science and Engineering B 2009; 159-160: 299-304.

12. Basore PA. IEEE Transactions on Electron Devices, 1990; 37: 337.

13. Hofstetter J, Fenning DP, Bertoni MI, Lelièvre JF, del Cañizo C, Buonassisi T. Impurity-to-efficiency simulator: predictive simulation of silicon solar cell performance based on iron content and distribution. Progress in Photovoltaics: Research and Applications 2011; 19: 487.

14. Shockley W, Read WT. Physics Reviews 1952; 87: 835; Hall RN. Physics Reviews 1952; 87; 387.

15. Coletti G, Bronsveld PCP, Kvande R, Geerligs LJ, Swanson CC at Workshop on solar grade silicon feedstock specification, Amsterdam, the Netherlands, 2008.

16. MacDonald D, Cuevas A. Reduced fill factors in multicrystalline silicon solar cells due to injectionlevel dependent bulk recombination lifetimes. Progress in Photovoltaics 2000; 8: 363. 
17. Istratov AA, Huber W, Weber ER. Applied Physics Letters 2004; 85: 4472.

18. Schmidt J, Krain R, Bothe K, Pensl G, Beljakowa S. Journal of Applied Physics 2007; 102: 123701.

19. Istratov AA, Hieslmair H, Weber ER. Applied Physics A: Materials Science and Processing 1999; 69: 13.

20. Paudyal BB, McIntosh KR, Macdonald DH. Journal of Applied Physics 2009; 105: 124510.

21. Coletti G, Geerligs LJ, Manshanden P, Swanson C, Riepe S, Warta W, Arumughan J, Kopecek R. Impact of iron and molybdenum in mono and multicrystalline float-zone silicon solar cells. Solid State Phenomena 2008; 131-133: 15-20.
22. Coletti G, Kvande R, Mihailetchi VD, Geerligs LJ, Arnberg L, Øvrelid EJ. Effect of iron in silicon feedstock on p- and n-type multicrystalline silicon solar cells. Journal of Applied Physics 2008; 104: 104913.

23. Swanson RM. Approaching the $29 \%$ limit efficiency of silicon solar cells. 20th European Photovoltaic Solar Energy Conference and Exhibition, Barcelona, Spain, 6-10 June, 2005.

24. Geerligs LJ, Macdonald D. Applied Physics Letters 2004 85: 4061; Coletti G, Kvande R, Mihailetchi VD, Geerligs LJ, Arnberg L, Øvrelid EJ. Journal of Applied Physics 2008; 104: 104913. 